\title{
Dust Grain Alignment in the Interstellar Medium
}

\author{
John E. Vaillancourt* and B-G Andersson \\ SOFIA Science Center, Universities Space Research Association, NASA Ames Research Center, \\ Moffett Field, CA, USA \\ E-mail: jvaillancourtesofia.usra.edu, bganderssonesofia.usra.edu
}

\section{Alex Lazarian}

University of Wisconsin - Madison, Madison, WI, USA

E-mail: alazarianefacstaff.wisc.edu

The first observations of interstellar polarization at visible wavelengths over 60 years ago were quickly attributed to the net alignment of irregular dust grains with local magnetic fields. This mechanism provides a method to measure the topology and strength of the magnetic field and to probe the physical characteristics of the dust (e.g., material, size, and shape). However, to do so with confidence, the physics and variability of the alignment mechanism(s) must be quantitatively understood. The description of the physical alignment mechanism has a long history with key contributions spanning decades; the last 15 years have seen major advances in both the theoretical and observational understanding of the problem. For example, it is now clear that the canonical process of paramagnetic relaxation, in which grain rotational components perpendicular to the magnetic field are damped out, is inadequate to align grains on the necessary timescales (compared to damping via collisions) for typical interstellar medium conditions. However, the modern theory of radiative alignment has been more successful; in this theory grains are aligned with respect to the magnetic field via photon-grain interactions that impart the necessary torques to the rotation axes of grains.

Here we highlight key observational tests of these alignment mechanisms, especially those involving spectropolarimetry of both dust extinction at near-optical wavelengths and dust emission at far-infrared through millimeter wavelengths. Observations in both these regimes can place limits on such grain aspects as their size and temperature. To date, most observations of the polarized emission have been in the densest regions of the interstellar medium where interpretation in terms of grain alignment models is complicated by regions containing embedded stars and a wide range of temperatures. Additionally, direct comparison of the optical extinction polarization $\left(A_{V} \lesssim 10\right.$ magnitudes) with dust emission polarization ( $A_{V} \lesssim 25$ magnitudes) has not been possible. Future observations with increased sensitivity and spectral coverage in the far-infrared may reach the lower extinction levels necessary to allow more definitive tests of grain alignment models.

The Life Cycle of Dust in the Universe: Observations, Theory, and Laboratory Experiments 18-22 November, 2013

Taipei, Taiwan

\footnotetext{
* Speaker.
} 


\section{Introduction}

Observations of polarized light toward celestial sources spanning ultraviolet to millimeter wavelengths are typically attributed to dust, be it interstellar, circumstellar, or interplanetary. The physical link between the observed polarization and dust was established by the earliest polarization measurements (1949), which were clearly correlated with interstellar extinction [1, 2]. At nearoptical wavelengths (UV, optical, and near-infrared) polarization arises from dichroic extinction of background starlight by dust, while at far-infrared and longer wavelengths the polarization arises from the thermal emission of the same dust, whose axes have different emission cross-sections.

In order to produce a net polarization, in either the emission or extinction cases, there must be a net alignment of axes within an interstellar grain population. This alignment is provided by local magnetic fields, where the smallest grain axis is aligned parallel to the field direction. The agreement between the inferred magnetic field orientation from optical and radio synchrotron polarization position angles $[3,4]$ provides direct observational support for the role of magnetic fields. However, despite decades of work, the physical mechanism by which grains become aligned with the fields is still uncertain. Magnetic fields as inferred from polarization have provided a key tool in studying the astrophysical effects of the fields on processes ranging from star-formation to the generation of turbulence in interstellar clouds. Hence a quantitative understanding of the alignment mechanism is clearly warranted, lest a wide range of work be invalidated. For example, one key question is whether grains are aligned in the same manner (or at all) in all physical conditions, and thus whether magnetic fields can be measured via polarization in all such conditions.

Here we briefly review some key aspects of grain alignment theory as they relate to existing and future tests. We further limit ourselves to tests requiring multi-wavelength observations. For more detailed discussions we direct interested readers to more comprehensive reviews [5-7].

\section{Alignment Mechanisms}

Alignment of grains with respect to magnetic fields requires at least two physical steps [8]. First the grain axis with the largest moment of inertia (the smallest axis) is aligned with the grain rotation axis. Secondly, the spin axis becomes aligned with the local magnetic field. A physical alignment model is generally considered successful if it can produce alignment on time-scales faster than the alignment can become disrupted by collisions with gas and other dust grains.

The first step in the alignment process occurs through an internal dissipation of energy that is minimized when the spin and inertial axes are parallel. Through a process known as the Barnett effect, a magnetization is induced in the paramagnetic grain due to its physical rotation and the existence of unpaired electrons in the solid. The largest component of the internal dissipation then arises from the interaction of the time-varying magnetic field (in grain coordinates) with the magnetization of the grain $[9,10]$. The initial model of paramagnetic dissipation [11] with modifications over the years remains the "textbook" mechanism most often cited to explain angular momentum alignment, the second alignment step. However, this mechanism has failed some key theoretical and observational tests, leaving the model of radiative torques as the most promising mechanism.

Paramagnetic relaxation, as first described by Davis \& Greenstein [11], arises from the dissipation of a grain's rotational energy components perpendicular to the ambient magnetic field. 
Paramagnetic alignment in the diffuse ISM will align grains on time-scales faster than disruption via gas-grain and grain-grain collisions only if 1) there is a temperature difference between the gas and dust populations that drives the grains to sufficient angular velocities [12]; and 2a) grain rotational energies are suprathermal ( $\gg k T)$ [9]; or 2b) grains contain superparamagnetic (e.g., ferromagnetic) inclusions [12]. Recent observations suggest that these latter conditions are not met in many regions of the ISM. For example, no correlations are observed between the amounts of solid iron in dust (as inferred from depletion measurements) and the level of observed polarization [13], as might be expected if superparamagnetic inclusions are required for grain alignment. Additionally, polarized lines of CO ice have been observed in molecular clouds [14], in regions where column densities are large enough that one expects near equilibrium between gas and dust temperatures, thereby severely limiting the ability of grains to reach suprathermal rotation rates [6].

The model of Radiative Alignment Torqes (RAT; [7, 15-18]) takes note of the fact that irregularly shaped grains have different cross-sections to left- and right-circularly polarized photons (i.e., have a non-zero net helicity) and that grains are exposed to an anisotropic radiation field. As the rotation axis of the magnetized grains precesses about the local magnetic field, the photons impart a net torque to the grains. On average, one torque component increases the grain rotation rate while another orthogonal component is in the direction which aligns the rotation axis with the magnetic field direction.

\section{Observational Tests}

The quantitative theory of RAT makes a number of clear observational predictions [6]. Specifically, 1) grains are aligned when exposed to an anisotropic radiation field of wavelength $\lambda \lesssim 2 a$, where $a$ is a characteristic grain radius; 2) the aligned-grain size distribution varies with radiation field color and intensity; 3) alignment efficiency varies with radiation field intensity; and 4) alignment efficiency depends upon the angle between the magnetic field and radiation anisotropy directions. In the remainder of this work we will highlight specific observational tests which can be performed using polarization spectra. As such, we will not further discuss point (4) other than to note that the relevant angle dependence has been observed [19].

\subsection{Optical \& Near-Infrared Tests}

The typical polarization spectrum of background starlight at optical wavelengths follows the empirical Serkowksi law, which is characterized by a maximum polarization at a wavelength $\lambda_{\max }$ in the range $\sim 0.4-0.8 \mu \mathrm{m}$ (see inset Fig. $1 b$ ). By comparing this spectrum to the standard wavelength-dependent extinction curve, Kim \& Martin [20] showed that large grains were more efficiently aligned than smaller grains. Specifically, they showed that the size distribution of grains responsible for the observed polarization had a small-size cut-off between 0.04-0.05 $\mu \mathrm{m}$ (e.g., the blue $A_{V}=0$ curve in Fig. 1a), while the total extinction data also requires smaller grains (e.g., [21]).

(1) This correlation between the shape of the optical polarization curve and the grain size distribution allows a number of additional observational tests. Firstly, we note that the fundamental prediction of RAT is that the most efficiently aligned grains are those of size $a \gtrsim \lambda / 2$. In the general ISM neutral hydrogen is expected to absorb all radiation shortward of the Lyman limit, $0.0912 \mu \mathrm{m}$. 

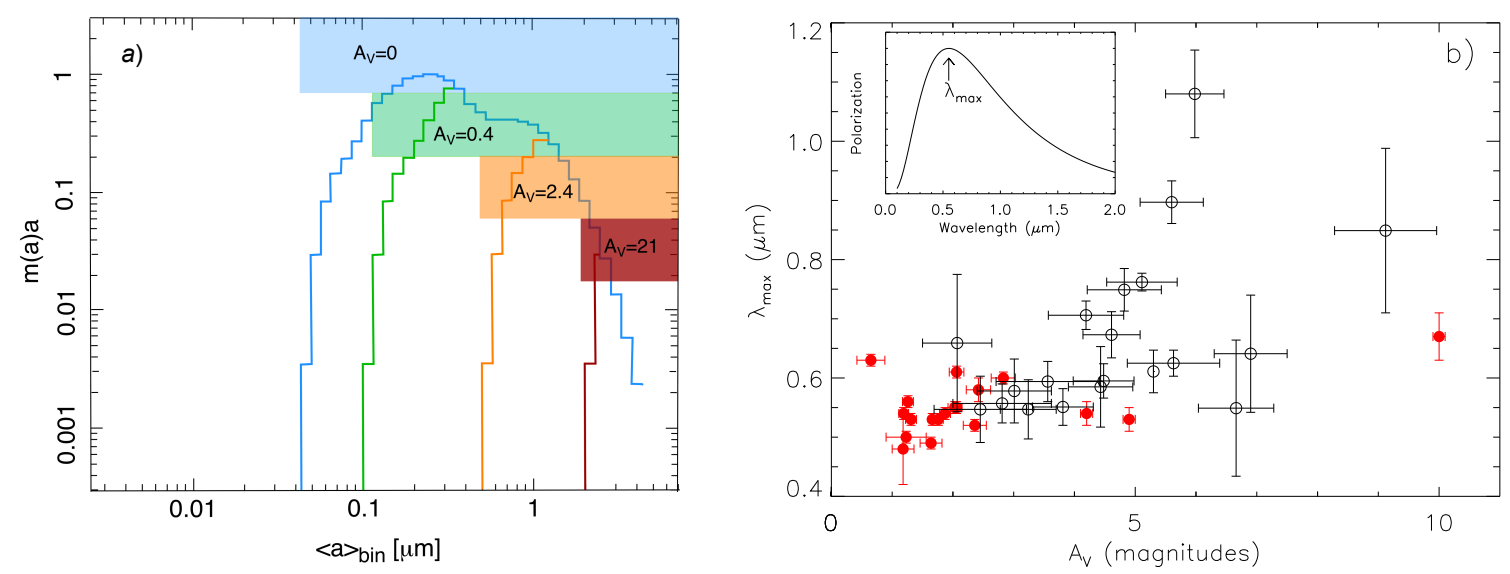

Figure 1: a) Example grain-size (mass) distributions for varying extinction levels $\left(A_{V}\right)$. Note that the upper size cut-off is the same for all curves. $b$ ) Measured values of the wavelength peak, $\lambda_{\max }$, in the near-optical polarization spectra as a function of line-of-sight extinction. Data shown here are towards stars in the Taurus molecular cloud from Whittet et al. (red points; [22]) and Vaillancourt et al. (in prep.; black points). The inset schematic shows a typical optical polarization (i.e., Serkowski) curve identifying the $\lambda_{\max }$ parameter.

Therefore, grains of radius $\lesssim 0.046 \mu \mathrm{m}$ should not be well aligned; this prediction is in excellent agreement with the observed grain size cut-off of 0.04-0.05 $\mu \mathrm{m}$ estimated by Kim \& Martin [20].

(2) When the aligning radiation field shifts to longer values, as might occur to light reddened via interstellar extinction, the RAT model predicts that the small grain-size cut-off should increase. Figure $1 a$ shows an example of the changing aligned grain distribution, as well as the increase in the mean size of aligned grains, as a function of extinction in the range $A_{V}=0-21$ magnitudes. The strong coupling between grain size and the observed wavelength of polarized radiation results in a shift in the spectrum to longer wavelengths, that is, an increase in $\lambda_{\max }$. The resulting correlation between $A_{V}$ and $\lambda_{\max }$ has been observed in a number of clouds (Fig. 1b; [22, 23]).

(3) Since RAT requires photons for grain alignment, it is reasonable to expect that alignment will be increased in regions of higher radiative intensity. Corresponding enhancements in background starlight polarization have been observed near embedded stars in Galactic clouds when compared to regions away from such sources [24]. Additionally, grains of similar composition exposed to different radiation fields will reach different equilibrium temperatures. Andersson \& Potter [23] have observed the expected correlation of grain color temperature (traced with imaging data at 60 and $100 \mu \mathrm{m}$ ) with alignment efficiency (as traced by $\lambda_{\max }$ ).

\subsection{Far-Infrared and Millimeter Tests}

As with optical measurements, polarized spectroscopy should provide the best tests of grain alignment at far-infrared through millimeter wavelengths. The best such measurements to date are summarized in Figure $2 a$. Most of the data are characterized by a polarization minimum somewhere in the range $100-850 \mu \mathrm{m}$, a shape which is not consistent with isothermal dust but rather requires some correlation between temperature and alignment efficiency [25, 26]. Instrument sensitivity currently limits observations to bright dense molecular clouds, although this has recently been extended to the diffuse ISM by experiments such as Planck [27], albeit with low spatial resolution ( $5^{\prime}$ for Planck at $\lambda>850 \mu \mathrm{m}$ compared to $10^{\prime \prime}-20^{\prime \prime}$ for ground-based work at $350-850 \mu \mathrm{m}$ ). 

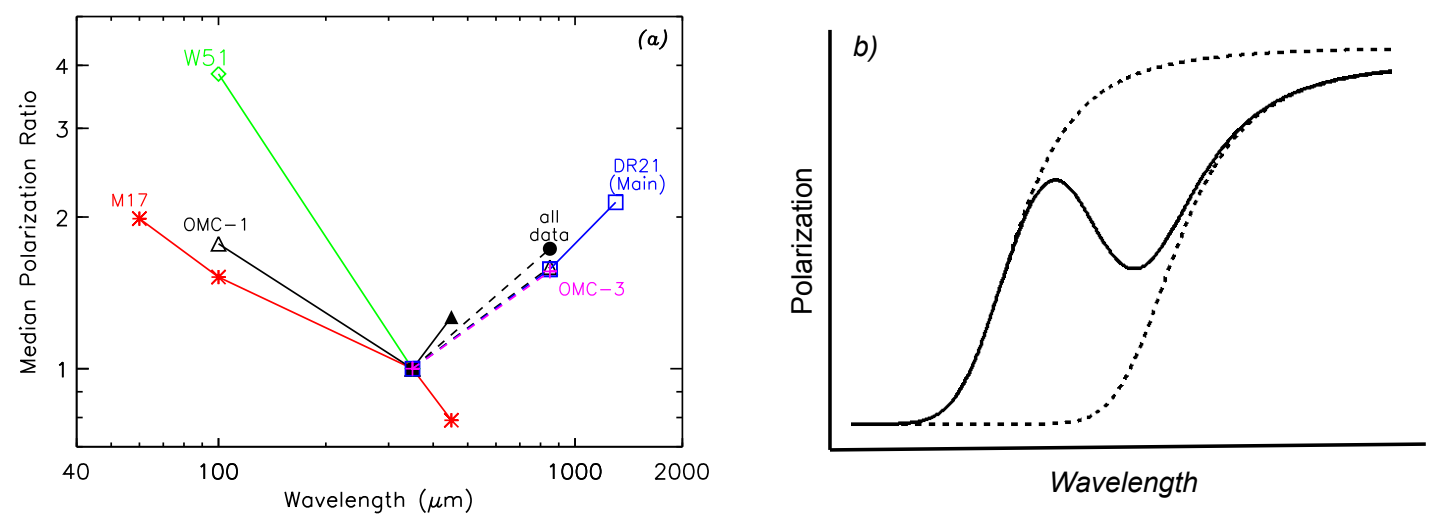

Figure 2: a) Median polarization ratio in various Galactic clouds, normalized at $350 \mu \mathrm{m}$ (e.g., [28, 29] and references therein). $b$ ) Schematic far-infrared $(\sim 10-1000 \mu \mathrm{m})$ polarization spectrum for a cloud with embedded stars. Dotted lines show expected spectra from two regions with different aligning radiation fields (both color and intensity), while the solid line is the mean spectrum.

One natural consequence of the RAT model is that large grains are more efficiently aligned than small grains [30]; this fact leads to variations in the aligned-grain size distribution (2). Additionally, when all grains are exposed to the same radiation field, one expects larger grains to be cooler since they will emit more efficiently. As a result, the warmer (smaller) grains are less efficiently aligned. Since the short-wavelength emission is dominated by these warmer, unaligned grains, the polarization spectrum is expected to rise with increasing wavelength (e.g., dashed lines in Fig. 2b; $[31,32])$. This model can explain the rising polarization spectrum observed longward of $350 \mu \mathrm{m}$, but cannot alone explain the falling spectra shortward of $350 \mu \mathrm{m}$.

In the dense molecular clouds observed to date, one cannot always isolate regions with different physical conditions (e.g., density, temperature, grain sizes, chemical composition, and radiation field intensity/color) but must instead integrate along the line-of-sight and within finite beam areas. For example, regions with embedded stars will have significantly different radiation fields from those without stars, resulting in a region with very warm dust and presumably highly efficient RATs. In isolation, such a region should also exhibit rising polarization spectra due to the correlation between grain size, temperature, and alignment. Observations encompassing regions with very different temperatures will exhibit significant separation of the basic spectral shape in wavelength-space (as shown by the two separate dashed spectra in Fig. 2b), resulting in complex total polarization spectra similar to those actually observed.

\section{Future Observations}

In the most general sense, the goal of future work is to observe polarization spectra over as wide a spectral range as possible and to do so in regions with a wide range of physical conditions to provide further quantitative tests for RAT. For example, does the $A_{V}-\lambda_{\max }$ correlation continue to higher extinction values and is it observed in many different clouds?

At far-infrared and millimeter wavelengths observations are needed to further characterize the polarization spectrum, which is very sparsely sampled in wavelength space (Fig. 2a). Tests of the RATs will require such spectral measurements in regions with different physical conditions. For 
example, increased spatial resolution $\left(\lesssim 10^{\prime \prime}\right)$ will separate of regions with and without embedded stars, allowing tests as a function of varying radiation intensity. Additionally, different models of grain composition predict different polarization spectra longward of $\sim 0.5 \mathrm{~mm}$, depending on whether or not carbonaceous grains are aligned [32]. Experiments designed to measure the CMB polarization have begun to explore this regime [27, 33].

\section{Acknowledgments}

The authors were partially supported by the NSF through grant AST-1109469. Partial support for JEV was also provided by the NASA Herschel Science Center through an award issued by JPL/Caltech.

\section{References}

[1] W. A. Hiltner, Polarization of Stellar Radiation. III. The Polarization of 841 Stars, ApJ, 114, 241 (1951).

[2] K. Serkowski, Correlation Between the Regional Variations in Wavelength Dependence of Interstellar Extinction and Polarization, ApJ, 154, 115 (1968).

[3] T. A. T. Spoelstra, Linear polarization of the galactic radio emission at frequencies between 408 and 1411 MHz. II - Discussion, A\&A, 135, 238 (1984).

[4] L. Page, G. Hinshaw, E. Komatsu, et al., Three-Year Wilkinson Microwave Anisotropy Probe (WMAP) Observations: Polarization Analysis, ApJS, 170, 335 (2007) [astro-ph/ 0603450 ].

[5] R. H. Hildebrand, Magnetic Fields and Stardust, QJRAS, 29, 327 (1988).

[6] B-G Andersson, Interstellar Grain Alignment - Observational Status, in Magnetic Fields in Diffuse Media, eds. A. Lazarian \& E. M. de Gouveia Dal Pino (2012), in press, astro-ph/1208 . 4393.

[7] A. Lazarian, Tracing magnetic fields with aligned grains, J. Quant. Spectros. Radiat. Transfer, 106, 225 (2007), [astro-ph/ 0208487 ].

[8] W. G. Roberge, Alignment of Interstellar Dust, in ASP Conf. Ser. 309, Astrophysics of Dust, eds. A. N. Witt, G. C. Clayton, \& B. T. Draine, 467, ASP, San Francisco (2004).

[9] E. M. Purcell, Suprathermal rotation of interstellar grains, ApJ, 231, 404 (1979).

[10] A. Lazarian \& B. T. Draine, Nuclear Spin Relaxation within Interstellar Grains, ApJ, 520, L67 (1999).

[11] L. J. Davis \& J. L. Greenstein, The polarization of starlight by aligned dust grains, ApJ, 114, 206 (1951).

[12] R. V. Jones \& L. J. Spitzer, Magnetic alignment of interstellar grains, ApJ, 147, 943 (1967).

[13] N. V. Voshchinnikov, T. Henning, M. S. Prokopjeva, \& H. K. Das, Interstellar polarization and grain alignment: the role of iron and silicon, A\&A, 541, A52 (2012) [astro-ph/1203.3677].

[14] J. H. Hough, D. K. Aitken, D. C. B. Whittet, A. J. Adamson, \& A. Chrysostomou, Grain alignment in dense interstellar environments: spectropolarimetry of the 4.67- $\mu \mathrm{m}$ CO-ice feature in the field star Elias 16 (Taurus dark cloud), MNRAS, 387, 797 (2008).

[15] A. Z. Dolginov \& I. G. Mytrophanov, Orientation of cosmic dust grains, Ap\&SS, 43, 257 (1976).

[16] B. T. Draine \& J. C. Weingartner, Radiative Torques on Interstellar Grains. II. Grain Alignment, ApJ, 480, 633 (1997).

[17] A. Lazarian \& T. Hoang, Radiative torques: analytical model and basic properties, MNRAS, 378, 910 (2007), arXiv: 0707.0886.

[18] T. Hoang \& A. Lazarian, Radiative torque alignment: essential physical processes, MNRAS, 388, 117 (2008), [astro-ph/0707.3645]. 
[19] B.-G. Andersson, O. Pintado, S. B. Potter, V. Straižys, \& M. Charcos-Llorens, Angle-dependent radiative grain alignment. Confirmation of a magnetic field - radiation anisotropy angle dependence on the efficiency of interstellar grain alignment, A\&A, 534, A19 (2011) [astro-ph/1109.3698].

[20] S.-H. Kim \& P. G. Martin, The size distribution of interstellar dust particles as determined from polarization: Spheroids, ApJ, 444, 293 (1995).

[21] J. S. Mathis, W. Rumpl, \& K. H. Nordsieck, The size distribution of interstellar grains, ApJ, 217, 425 (1977).

[22] D. C. B. Whittet, P. A. Gerakines, J. H. Hough, \& S. S. Shenoy, Interstellar extinction and polarization in the Taurus dark clouds: The optical properties of dust near the diffuse/dense cloud interface, ApJ, 547, 872 (2001).

[23] B.-G. Andersson \& S. B. Potter, Observational Constraints on Interstellar Grain Alignment, ApJ, 665, 369 (2007) [astro-ph/ 0705.1306 ].

[24] D. C. B. Whittet, J. H. Hough, A. Lazarian, \& T. Hoang, The Efficiency of Grain Alignment in Dense Interstellar Clouds: a Reassessment of Constraints from Near-Infrared Polarization, ApJ, 674, 304 (2008) [astro-ph/0711.2536].

[25] R. H. Hildebrand, J. L. Dotson, C. D. Dowell, D. A. Schleuning, \& J. E. Vaillancourt, The Far-Infrared Polarization Spectrum: First Results and Analysis, ApJ, 516, 834 (1999).

[26] J. E. Vaillancourt, Analysis of the Far-Infrared/Submillimeter Polarization Spectrum Based on Temperature Maps of Orion, ApJS, 142, 53 (2002).

[27] V. Guillet, Planck First Polarization Results - Comparison of dust polarized emission at $353 \mathrm{GHz}$ with starlight polarization, in The Life Cycle of Dust in the Universe: Observations, Theory, and Laboratory Experiments, POS (LCDU2013) 055.

[28] J. E. Vaillancourt \& B. C. Matthews, Submillimeter Polarization of Galactic Clouds: A Comparison of $350 \mu \mathrm{m}$ and $850 \mu \mathrm{m}$ Data, ApJS, 201, 13 (2012).

[29] L. Zeng, C. L. Bennett, N. L. Chapman, D. T. Chuss, I. Jimenez-Serra, G. Novak, \& J. E. Vaillancourt, The Submillimeter Polarization Spectrum of M17, ApJ, 772, 29 (2013) [astro-ph/1306.3259].

[30] J. Cho \& A. Lazarian, Grain Alignment by Radiation in Dark Clouds and Cores, ApJ, 631, 361 (2005).

[31] T. J. Bethell, A. Chepurnov, A. Lazarian, \& J. Kim, Polarization of Dust Emission in Clumpy Molecular Clouds and Cores, ApJ, 663, 1055 (2007) [astro-ph/ 0611324 ].

[32] B. T. Draine \& A. A. Fraisse, Polarized Far-Infrared and Submillimeter Emission from Interstellar Dust, ApJ, 696, 1 (2009) [astro-ph/0809.2094].

[33] E. M. Bierman, T. Matsumura, C. D. Dowell, et al., A Millimeter-Wave Galactic Plane Survey With The BICEP Polarimeter, ApJ, 741, 81 (2011). 